\title{
P02-174
}

\section{MEMORY DISORDER IN PERSONS WITH ASPERGER SYNDROME}

\section{Milacic Vidojevic}

Faculty for Special Education and Rehabilitation, Beograd, Serbia

Aim: Asperger syndrome (AS) is a term used to mark a subgroup of autism with no delay in language or cognitive development. Research reveal language and cognitive impairments in AS (uneven profile of abilities on Wechsler intelligence scales, pragmatic deficits, receptive speech, narrow attention and difficulty in shifting attention, etc). The objective of this study is to examine episodic memory of children with AS. 12 children with AS age from 7-16 years old, 11 males, 1 female participated in the study. All children had an IQ in the normal range.

Method: Buschke Selective Reminding Test was used in the present study as a measure of episodic memory suitable for the age range of the children in the study.

Results: scores gained by the children with AS comparing to the scores of the normally developing children indicate deviation on the measures of recall, long term storage, consistent long term retrieval and increased number of intrusions. Only 3 subjects scored within normal limits. Information produced on immediate recall was not lost over 30-minute delay.

Conclusion: results shows dominant problem in organization of stored information. 